\title{
Subject Index Vol. 32, 1995
}

N-Acetyl-ß-glucosaminidase 75 Adrenergic activity 182 Affective disorders 1,13 Age 1

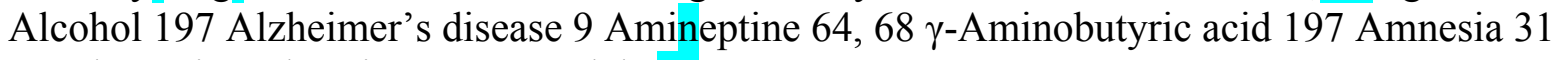

Amphetamine, chronic 149 Amygdala 31

Anorexia nervosa, binge-eating/purging type 64

Anxiety 182

Anxiolytics 156

Apomorphine test 19

Attention processes 156

Autism 120

Benzodiazepines 31 Blood electrolytes 13 pressure 182

Brofaromine 23

Bulimia nervosa 68

Buspirone 156

Carbohydrate-deficient transferrin 192 Carnitine 132

Catecholamine synthesis 124 Chaos theory 45 Cholinergic activity 182 Chronic alcoholism 192 fatigue syndrome 132,175

Cigarettes 161

Cognitive-behavioral therapy 59, 64,68 Complex demodulation 182 Correlation exponent 45

Dementia 81 Deoxyglucose 149 Depression 13, 182 Diazepam 31, 106,156 Disengagement, attention 156 Dopamine 19, 115, 197 Driving 161 Drug postulate 197 Dysthymia 128

Electroencephalography 81,214 Electrophysiology 208 Erythrocytes 13 Event-related potentials 52 
Extended haplotype 120 Extraversion 197

Fatigue 132,175 Fluoxetine 59, 64 Fluvoxamine 68

Generalized anxiety disorder 75 Global frequency analysis 45

Heart rate 182

HistamineHi receptor antagonists 214

HLA 120

5-Hydroxyindoleacetic acid 75

Imipramine 23 Information processing 72

Joan of Arc 79

Learning avoidance 31 Lithium 115

Liver function studies 192 Locomotor activity 149

Magnesium 13

transport 13 Major depression 19,23

depressive disorder 128

histocompatibility complex 120 Memory 31

impairment, epilepsy 37 Mental health 98 Mitochondria 175 Mizolastine 214 Monitoring abstinence 192 Mood 98

Muscimol 124

Neuroimaging 139

Neuroleptics 115

Neuronal activity 52

Nicotine 161

Normalized slope descriptors 45

Nortriptyline 59

Nucleus accumbens 149

Obsessive-compulsive disorder 139

P300 72

- $\quad$ amplitude 171

Performance 197

Pharmacoelectroencephalography 106,166

223

Pharmacotherapeutic treatment, memory 37

Picrotoxin 124

Platelet 9

Posttraumatic stress disorder 72

Psychometry 81

Psychomotor performance 161

Psychoses 1,115

Pyridoxine 98

Quantitative electroencephalography 139

Rating scales 81 Respiration 182 Riboflavin 98

Sabeluzole 37

Saccadic eye movements 156 - reaction time 156 Schizophrenia 1, 171 Sedation 214 Sensitization 149 


\section{Serotonin 9}

\section{reuptake inhibitors 115}

synthesis 124

Single-photon emission computerized tomography 139 Sleep EEG 1,45,128 Sleep-wakefulness cycle 214 Smoking 161 Sodium valproate 124

Tardive dyskinesia 115 Temporal lobe tuberculoma 79 Thiamin 98

Time domain descriptors 45 Trait marker 171 Tuberculosis 79

Visual system 208 Vitamin status 98

$$
\text { supplementation } 98
$$

Washout period 19 
